\title{
Welcome to the Journal of Surveillance, Security and Safety: A New Open-Access Scientific Journal
}

\author{
Xiaofeng Chen \\ School of Cyber Engineering, Xidian University, Xi'an 710126, Shaanxi, China. \\ Correspondence to: Prof. Xiaofeng Chen, School of Cyber Engineering, Xidian University, Xi'an 710126, Shaanxi, China. \\ E-mail:xfchen@xidian.edu.cn
}

\begin{abstract}
How to cite this article: Chen X. Welcome to the Journal of Surveillance, Security and Safety. A New Open-Access Scientific Journal. J Surveill Secur Saf2020;1:102-5. http://dx.doi.org/10.20517/jsss.2020.26
\end{abstract}

Received: 23 Sep 2020 Accepted: 23 Sep 2020 Published: 29 Sep 2020

Academic Editor: Xiaofeng Chen Copy Editor: Cai-Hong Wang Production Editor: Jing Yu

\section{INTRODUCTION}

This journal has been anxiously awaited by those interested in the security and safety problems associated with artificial intelligence, the blockchain, databases, cloud computing, multimedia, wireless networks, IoT, and other computer science and cryptography technologies.

In recent years, security threats faced by new technologies are emerging without end, while the security requirements of traditional technologies are increasing. Interest in these areas has grown rapidly, mainly including the security issues from the perspectives of AI, data, network, computing, cryptography, access control, industries, policies, models, etc. The deeper is the awareness of private data that people have, the higher is their need for application security.

Explosive growth in the number and scale of machine learning models, requiring robustness in their training and testing periods against adversarial attacks, is one of the most striking characteristics of the current technological landscape about artificial intelligence. The expansion of research related to both machine learning-based attacks and their interpretation has driven the rapid growth of the research area in secure machine learning models. The urgent need for security research is not a unique trend in a certain field, and tricky challenges regarding security issues also appear in other popular areas, e.g., the blockchain, where multiple system components, such as consensus mechanism and smart contract, have been found susceptible to malicious attacks that destroy the credibility of the system.

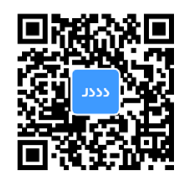




\section{JOURNAL OF SURVEILLANCE, SECURITY AND SAFETY: WHY A NEW JOURNAL?}

While related Special Sections of existing journals have appeared regularly, research results in the fields of surveillance, security, and safety have been published in many different journals, creating a somewhat dispersed audience. In response, the Journal of Surveillance, Security and Safety (JSSS) has been badly needed as a platform for publishing research results in the foundations, methods, and mechanisms, with surveillance, security, and safety tending to have been to be treated separately.

Surveillance involves relevant observation methods that aim at preventing or detecting crime behavior. Security, with its classic foundations in cryptography, involves issues related to data and privacy protection, intrusion detection, authentication, protocols, and reliable transaction, as well as other security-related fields. Safety is primarily defined as including the means designed to prevent inadvertent or hazardous operation. I am also certain that this publication will be of interest to everyone working in the general area of surveillance, security, and safety.

The journal is the result of the dedication of many individuals who are selflessly willing to put in long hours of work in an attempt to give back to the community. We owe thanks to those who completed the initial work and those who will follow through in the next few years.

\section{WHAT IS SPECIAL IN THE FIRST ISSUE?}

The journal has an impressive opening in the first issue. These papers illustrate various studies that can advance our understanding of surveillance, security, and safety when considered in a forum.

Shmidt et al. ${ }^{[1]}$ in "Learning and unlearning from disasters: an analysis of the Virginia Tech, USA shooting and the Lion Air 610 Airline crash" provide an analysis about organizational learning and theories of learning from failures. This work attempts to stimulate organizational learning and improve organizational processes to mitigate disasters from happening again. I expect this work to continue and extend the proposed methodology to other cases in the fields of surveillance, security, and safety.

$\mathrm{Xu}$ et al. ${ }^{[2]}$ s "Big data analytics of crime prevention and control based on image processing upon cloud computing" presents a cloud computing-based image processing technology to identify individual crimes and subject segmentation, which uses statistical methods to collect the characteristics of criminal behavior, addressing the issues of non-real-time observation of criminal behavior.

In "A survey of domain name system vulnerabilities and attacks", Kim and Reeves ${ }^{[3]}$ efficiently analyzed the vulnerabilities of DNS and four categories of representative DNS attacks. I expect the defense mechanisms introduced in this paper will motivate greater participation in this effort.

"Stereo storage structure assisted one-way anonymous auditing protocol in e-health system" by Jiang et al. ${ }^{[4]}$ examines the challenges of the design of the storage structure in the cloud and the data integrity verification in the medical environment with clients' privacy protection. A novel stereo storage structure-assisted oneway anonymous auditing protocol in the e-health system is proposed, which can implement the storage and fast search of medical data.

Salmani and Barker ${ }^{[5]}$ s "Leakless Privacy-Preserving Multi-keyword Ranked Search over Encrypted Cloud Data" presents an efficient encrypted cloud data search service that employs the secure inner product similarity and chaining encryption notion and addresses the problem of leakless privacy-preserving multikeyword ranked search over encrypted cloud data.

Future issues will contain additional papers solicited for the first issues and the latest solicited papers of the journal. These papers will continue this trend, that is the first issue begins with high hope. 


\section{WHAT IS THE SCOPE OF JSSS?}

As an international and interdisciplinary scholarly open-access journal, Journal of Surveillance, Security and Safety publishes original research articles, reviews, and communications that offer substantially new insights into the variety of theoretical, methodological, epistemological, empirical, and practical issues reflected in the field of information security, cyber security, machine learning, emerging technologies, and their applications. Papers are solicited from, but not limited to, the following topics:

- AI-based surveillance and security

- Privacy protection based on machine learning

- Security of machine learning algorithms

- Deep learning for attack and defense

- Database security

- Data-driven cybersecurity incident prediction

- Big data security

- Cloud/fog computing security

- Outsourcing and crowdsourcing security

- Security and privacy in pervasive/ubiquitous computing

- Cyber-physical systems security

- Security, privacy, and resilience in critical infrastructures

- Multimedia security

- Wireless network security

- Social networks and IoT security

- Information hiding, forensics, and security

- Theory and applications of cryptography

- Identity management, authentication, and access control

- Security policies, models, and architectures

- Electronic commerce security

- Blockchain and finance security

- Intrusion detection

- Phishing and spam prevention

- Biometrics

- Regulation of the security industry

- Risk analysis, security measures, and management

- Evaluations of Security Measures

This journal aspires to provide a venue to support constructive communications across different related areas of security. Occasionally, Special Sections on unique topics of high interest will be organized, receiving more exposure to readers by being presented alongside relevant articles. We welcome your suggestions for special issues and appropriate Guest Editors.

I warmly welcome the participation of all researchers interested in this challenging and compelling field. This inaugural journal is the realization of the long-term desire of many in the computer industry for surveillance, security, and security issues. I hope you enjoy it and support us with the launch of this new and exciting open-access journal that will help us to shape a safer information age.

\section{DECLARATIONS}

\section{Authors' contributions}

The author contributed solely to the article. 


\section{Availability of data and materials}

Not applicable.

\section{Financial support and sponsorship}

The author has not declared a specific grant for this editorial from any funding agency in the public, commercial, or not-for-profit sectors.

\section{Conflicts of interest}

The author declared that there are no conflicts of interest.

\section{Ethical approval and consent to participate}

Not applicable.

\section{Consent for publication}

Not applicable.

\section{Copyright}

(c) The Author(s) 2020.

\section{REFERENCES}

1. Shmidt B, Labib A, Hadleigh-Dunn S. Learning and unlearning from disasters: an analysis of the Virginia Tech, USA shooting and the Lion Air 610 Airline crash. J Surveill Secur Saf 2020;1:1-15.

2. Xu Z, Cheng C, Sugumaran V. Big data analytics of crime prevention and control based on image processing upon cloud computing. J Surveill Secur Saf 2020;1:16-33.

3. Kim TH, Reeves D. A survey of domain name system vulnerabilities and attacks. J Surveill Secur Saf 2020;1:34-60.

4. Jiang LH, Wang C, Shen J. Stereo storage structure assisted one-way anonymous auditing protocol in e-health system. J Surveill Secur Saf 2020;1:61-78.

5. Salmani K, Barker K. Leakless privacy-preserving multi-keyword ranked search over encrypted cloud data. J Surveill Secur Saf 2020;1:79-101. 